\title{
Effects of 12 months of exercise training on salivary secretory lgA levels in elderly subjects
}

\author{
T Akimoto, Y Kumai, T Akama, E Hayashi, H Murakami, R Soma, S Kuno, I Kono
}

Br J Sports Med 2003;37:76-79

See end of article for authors' affiliations

......................

Correspondence to: Dr Akimoto, Department of Life Sciences, Graduate School of Arts and Sciences, University of Tokyo, 3-8-1 Komaba, Meguro, Tokyo 153-8902, Japan;

axi@idaten.c.u-tokyo.ac.jp

Accepted 11 April 2002
Background: The immune system declines in efficiency with advancing age, making the elderly less resistant to pathogenic microorganisms. Upper respiratory tract infection (URTI) is a common illness. Recent studies have shown that suppression of secretory immunoglobulin $A(S \operatorname{SA})$ is associated with increased incidence of URTI.

Objective: To assess the effect of exercise on salivary SIgA in elderly subjects.

Methods: Forty five elderly subjects (18 men, 27 women; mean (SD) age 64.9 (8.4) years) performed both 60 minute resistance and 60 minute moderate endurance training a week for 12 months. Saliva samples were obtained before training, and at four and 12 months during the training period. Salivary $\mathrm{S} \lg \mathrm{A}$ concentrations were measured by enzyme linked immunosorbent assay, and the SIgA secretion rate was calculated.

Results: SlgA concentrations before training, and at four and 12 months during training were 24.7 (14.4), 27.2 (14.2), and 33.8 (18.5) $\mathrm{gg} / \mathrm{ml}$ respectively. SlgA secretion rates were 29.5 (26.0), 33.8 (27.2) and $46.5(35.1) \mathrm{\mu g} / \mathrm{min}$ respectively. The results indicate that both the concentration and secretion rate of $\operatorname{SlgA}$ significantly $(p<0.01)$ increased during 12 months of exercise in these elderly subjects.

Conclusion: Regular moderate exercise seems to enhance mucosal immune function in elderly subjects. t $\mathrm{t}$ is generally believed that immune function declines with age, and, compared with young people, the elderly are less resistant to pathogenic microorganisms. This may be explained by the decreased function of, or loss of regulation of, the immune system during aging. ${ }^{1}$ This age related decline in the regulation of immune function is also probably linked to increased incidence of malignancy, infectious disease, and autoimmune disorders. ${ }^{2}$ Respiratory infections, such as pneumonia and influenza, including upper respiratory tract infection (URTI), are particularly common and serious illnesses among the elderly.

Several investigators have examined endurance athletes who appear to be at high risk of contracting URTI as a result of high volume training. ${ }^{4-6}$ The results show a high incidence of URTI after competition in distance runners compared with similarly trained but non-competing runners $^{7}$ and age matched non-runners. ${ }^{8}$ Nieman ${ }^{9}$ has modelled a relation between exercise and URTI in the form of a " $\mathrm{J}$ " curve. This model suggests that, although the risk of URTI may decrease below that of a sedentary person during moderate exercise training, the risk may rise during periods of high intensity exercise.

Recent studies have shown that suppression of salivary secretory immunoglobulin A (SIgA) is associated with increased incidence of URTI in elite athletes. ${ }^{10}$ SIgA is the predominant immunoglobulin in secretions of the mucosal immune system. ${ }^{11}$ It is found in the saliva, intestinal secretions, bronchoalveolar lavage fluid, urine, tears, and other mucosal fluids. It inhibits attachment and replication of pathogenic microorganisms, preventing colonisation by these pathogens; it is also capable of neutralising toxins and viruses. ${ }^{611-13}$

Resistance exercise enhances muscle strength, muscle size, and resting metabolic rate in young adults, and recent reports have documented similar positive adaptations in elderly

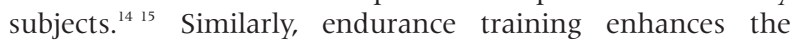
aerobic capacity of elderly subjects. These findings indicate that exercise has many effects in elderly subjects. ${ }^{16}$ Exercise is thought to modulate immune function. ${ }^{97} \mathrm{~A}$ few studies have examined the effect of exercise on the cellular immune function of elderly subjects. ${ }^{18-20}$ Nieman et al ${ }^{2}$ showed that there was no change in natural killer (NK) and $\mathrm{T}$ cell function in elderly subjects after 12 weeks of moderate cardiorespiratory exercise, but that highly conditioned elderly women had superior NK and T cell function compared with sedentary age matched women. In the study of Crist et al, elderly women engaged in 16 weeks of endurance training; the exercising group developed increased resting NK activity compared with the sedentary controls. Furthermore, Shinkai et $a^{20}$ have shown that habitual endurance exercisers retain a higher level of $\mathrm{T}$ cell function than sedentary people in old age in a cross sectional survey. However, no studies have investigated the effects of exercise on mucosal immune function, such as salivary SIgA secretion in elderly subjects.

In this study, we examined the influence of exercise on mucosa related immunity in elderly subjects. We designed a 12 month clinical study to measure the effects of single 60 minute resistance and 60 minute endurance training sessions a week on salivary SIgA levels. The aim was to determine whether 12 months of moderate combined exercise training could enhance salivary SIgA in elderly subjects.

\section{METHODS}

\section{Subjects}

Forty five sedentary, but healthy elderly subjects (18 men, mean (SD) age 67.3 (3.6) years; 27 women, mean (SD) age 63.7 (6.9) years) volunteered to participate in a 12 month

Abbreviations: URTI, upper respiratory tract infection; SlgA, secretory immunoglobulin $\mathrm{A} ; \mathrm{V}_{2} \mathrm{MAX}$, maximal oxygen uptake; $\mathrm{NK}$, natural killer; $H R$, heart rate 
training programme. Subjects were recruited by the corporation of Taiyo village officer. Potential subjects were given a detailed explanation of the risks, stress, and potential benefits of the study before they signed an informed consent form. The study protocol was in accordance with the policy statement of the institutional review board of the University of Tokyo. All subjects had passed a complete medical examination within the past year and received written permission from a specialist sports doctor to be included in the study. In addition, subjects that met the exclusion criteria of the American College of Sports Medicine ${ }^{22}$ were not allowed to participate. No subjects were receiving treatment known to affect immune function. There were no patients with allergies or acute infections.

\section{Study design}

The study was designed to examine the combined effects of resistance exercise and endurance exercise. Saliva samples were collected from subjects on three occasions (November 1997, March 1998, November 1998) during a 12 month moderate exercise training programme. Saliva samples were collected at 0830-0930 hours after the subjects had fasted for at least nine hours, had avoided exercise for 12 hours, and had rested in a seated position for at least 15 minutes.

\section{Exercise}

The subjects trained twice a week at a fitness club, once in a group session for resistance training and once for endurance training. About 10 minutes of stretching preceded each training session. Both training sessions were supervised and conducted by an experienced instructor who was also responsible for the heart rate (HR) measurement.

The resistance training session comprised two sets of inner thigh, rowing, squat, trunk curl, chest press, and back extension exercises (8-15 repetitions), using a combination of free weights and an exercise machine and 20-30 minutes on a cycle ergometer at $60 \%$ maximal HR. Upper and lower body exercises were alternated to minimise fatigue, and a sufficient rest interval between the two sets was allowed.

The endurance training programme comprised flexibility and aerobic exercises, such as step exercise with music and ball games. The target HR was calculated using the HR reserve method proposed by Karvonen et $a l^{23}$ such that during the activity the HR was kept below $60 \%$ of HR reserve.

\section{Estimation of individual $\mathrm{VO}_{2} \mathrm{MAX}$}

Individual $\mathrm{VO}_{2} \operatorname{MAX}$ was estimated by the method of Astrand with some modification. ${ }^{24}$ Each subject sat on a cycle ergometer and performed a three stage incremental ergometer exercise for 12 minutes. Exercise intensity was determined according to age and sex. During the exercise, mean HR was measured before the end of each stage.

\section{Saliva collection}

Saliva samples were collected as described previously. ${ }^{25}$ Briefly, timed whole mixed saliva samples were collected after the mouth had been rinsed thoroughly with distilled water. Saliva production was stimulated by chewing a sterile cotton swab (Salivette; Sersted, Vümbrecht, Germany) at a frequency of $60 / 60$ seconds, and saliva was separated from the cotton by centrifugation at $3000 \mathrm{rpm}$. After measurement of the sample volume, saliva samples were frozen at $-80^{\circ} \mathrm{C}$ and stored until the end of the study period.

\section{Assays}

Salivary SIgA concentrations were measured by enzyme linked immunosorbent assay (ELISA) as described previously. ${ }^{25}$ Briefly, a 96 well microtitre plate (Immulon II; Dynex Technologies, Chantilly, Virginia, USA) was coated with rabbit anti-(human secretory component) IgG fraction (MBL, Nagoya, Japan) overnight at $4^{\circ} \mathrm{C}$. After the addition of $250 \mu \mathrm{l}$ phosphate buffered saline containing $1 \%$ bovine serum

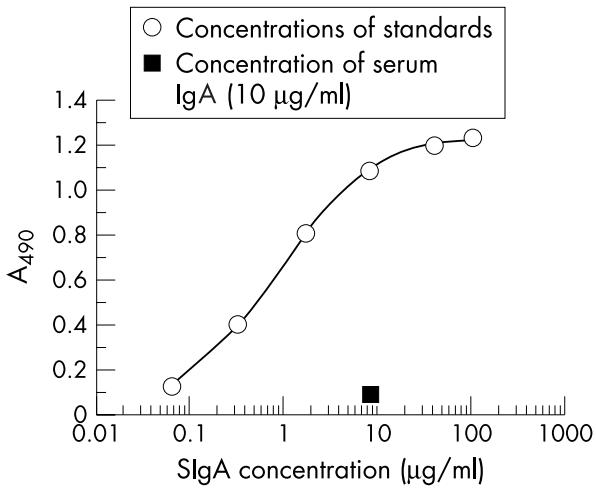

Figure 1 Standard enzyme linked immunosorbent assay curve for measurement of salivary secretory immunoglobulin $\mathrm{A}(\mathrm{S} \lg \mathrm{A})$.

albumin (Sigma, St Louis, Missouri, USA), wells were blocked for two hours. Saliva samples were thawed, centrifuged at $10000 \mathrm{rpm}$ for five minutes, and diluted (1:20) with phosphate buffered saline containing $1 \%$ bovine serum albumin; $100 \mu \mathrm{l}$ of each was added and the mixture incubated for one hour. Using purified human SIgA (Organon Teknika, Durham, North Carolina, USA), known concentrations of SIgA were also plated to establish standard values. After the plate had been washed with phosphate buffered saline/Tween, goat Fab' anti-IgA conjugated with horseradish peroxidase (MBL) was added to the plate and the mixture incubated for one hour. After washing, substrate solution was added and the colour intensity produced after 15 minutes was measured by a microplate reader (Bio-Rad Laboratories, Hercules, California, USA) at $490 \mathrm{~nm}$. All samples were assayed in duplicate, and the average of absorbance values was used as the representative value. Regression analysis using the relation of standard SIgA concentrations and amount of absorbance (nm) was used to interpolate the concentration of SIgA in the samples. Figure 1 shows a typical standard curve. To avoid interassay variability, all samples from each subject were assayed on the same plate. The interassay coefficient of variation of the method, based on analysis of 82 duplicate samples, was $6.2 \%$. Concentrations of total protein in the saliva were measured by using the method of Bradford (Bio-Rad Laboratories).

\section{Statistical analysis}

For analysis of SIgA levels, data were expressed in two forms: (a) absolute concentration of SIgA $(\mu \mathrm{g} / \mathrm{ml})$; (b) SIgA secretion rate $(\mu \mathrm{g} / \mathrm{min})$, or the total amount of SIgA appearing on the mucosal surface per unit time. SIgA secretion rate was calculated by multiplying absolute SIgA concentration $(\mu \mathrm{g} / \mathrm{ml})$ by saliva flow rate $(\mathrm{ml} / \mathrm{min})$, which was calculated by dividing the total volume of saliva obtained in each sample $(\mathrm{ml})$ by the time taken to produce the saliva sample (minutes). ${ }^{26}$ Salivary SIgA concentration, saliva flow rate, and SIgA secretion rate were analysed separately by one way analysis of variance with repeated measures to determine the effect of training. When one way analysis of variance showed significant effects, a Fisher's post hoc test was performed. For all analyses, $\mathrm{p}<0.05$ was accepted as significant.

\section{RESULTS}

\section{Estimated $\mathrm{VO}_{2} \mathrm{MAX}$}

The estimated $\mathrm{VO}_{2} \operatorname{Max}$ had significantly increased after 12 months of moderate exercise training (31.4 (5.5) v 32.5 (6.8) $\mathrm{ml} / \mathrm{min} / \mathrm{kg}$; mean (SD); $\mathrm{p}<0.05$ ).

\section{Saliva concentration of total proteins}

Mean concentrations of total proteins before training, and after four and 12 months of training were $0.72(0.16), 0.73$ 


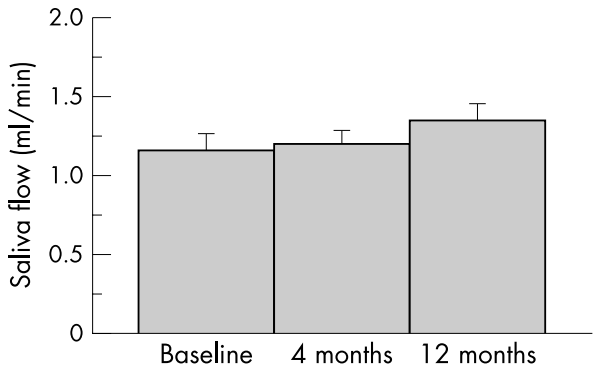

Figure 2 Rate of saliva flow before training and after four and 12 months of moderate exercise training. Values are mean (SD).

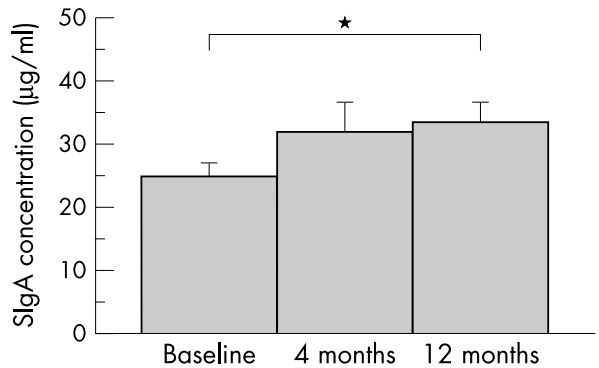

Figure 3 Concentration of salivary secretory immunoglobulin A $(S \lg A)$ before training and after four and 12 months of moderate exercise training. Values are mean (SD). ${ }^{*} p<0.05$.

(0.09), and $0.72(0.14) \mu \mathrm{g} / \mathrm{ml}$ respectively (fig l). Therefore total protein concentration did not change during the study.

\section{Saliva flow rate}

Samples of stimulated saliva were collected at rest before training, and after four and 12 months of training. Mean saliva flow rates before training, and at four and 12 months were $1.17(0.56), 1.20(0.61)$, and $1.35 \quad(0.61) \mathrm{ml} / \mathrm{min}$ respectively (fig 2 ). The rate had increased slightly after four and 12 months of training, but the difference was not significant.

\section{SIgA concentration}

Mean SIgA concentrations before training, and after four and 12 months of training were 24.7 (14.4), 27.2 (14.2), and 33.8 (18.5) $\mu \mathrm{g} / \mathrm{ml}$ respectively (fig 3). Although SIgA concentration had increased after four months of training, this was not significant. However, after 12 months of training, the SIgA concentration was significantly higher than at baseline $(p<0.05)$.

\section{SIgA secretion rate}

The calculated mean SIgA secretion rates before training, and after four and 12 months of training were 29.5 (26.0), 33.8 (27.2), and 46.5 ( 35.1$) \mu \mathrm{g} / \mathrm{min}$ respectively (fig 4 ). The rate was significantly higher after both four $(p<0.05)$ and 12 months of training $(\mathrm{p}<0.01)$ compared with baseline.

\section{DISCUSSION}

Our study provides evidence that regular moderate exercise increases salivary SIgA levels in elderly subjects. This result suggests that moderate exercise may improve the mucosal immunity of the elderly. To our knowledge, the present data are the first to show that mucosal immune function (salivary SIgA levels) is enhanced in elderly subjects after 12 months of moderate exercise. However, our study did not include a control group, and the exact contribution of each exercise type (resistance training and endurance training) could not be separated; rather the results should be viewed as the effect of moderate exercise on mucosal immune function in the elderly.

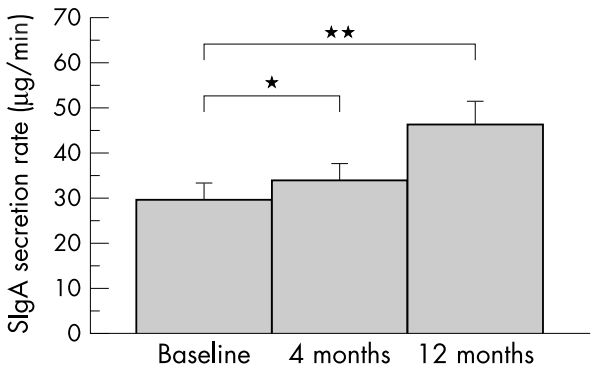

Figure 4 Rate of secretion of secretory immunoglobulin $\mathrm{A}(\mathrm{S} \lg \mathrm{A})$ before training and after four and 12 months of moderate exercise training. Values are mean (SD). ${ }^{*} p<0.05 ;{ }^{* *} p<0.01$.

Mackinnon and Jenkins ${ }^{26}$ reported that resting salivary SIgA levels in athletes had not changed after eight weeks of interval training. One explanation for the difference from our results may be differences in baseline characteristics of the participating subjects-for example, age and endurance capacity. Reviews of reports by several groups have suggested that the effects of exercise on the immune system depend on the level of fitness of the participating subjects and the intensity and duration of the exercise, such that exercise in sedentary subjects generally induces improvement in immune parameters. ${ }^{27}$ Our study may support this view. It is possible that the time period used by Mackinnon and Jenkins ${ }^{26}$ was too short to influence resting salivary SIgA levels.

Saliva is a complex mixture of secretions from the parotid, submandibular, and sublingual glands and also from many other smaller glands. These glands are the most important source of SIgA in the upper respiratory tract. ${ }^{11}$ SIgA plays an important part in immune protection at the mucosal surface by providing specific antibodies in response to pathogens, and forms an exclusion barrier at the mucosal surface to prevent antigen entry. ${ }^{6}$ The lack of non-specific SIgA at the mucosal surface or the inability to produce specific SIgA can lead to increased risk of infection, as in cases of IgA deficiency. ${ }^{28}$

Gleeson $e t$ a ${ }^{10}$ provided evidence that reduced levels of salivary SIgA are associated with increased frequency of episodes of URTI. Mackinnon and Jenkins ${ }^{26}$ suggested that at least part of the increased susceptibility to URTI in athletes may be due to reduction of SIgA output resulting from both decreased SIgA secretion and inhibition of salivary flow that occurs briefly after intense exercise. Miletic et al ${ }^{29}$ reported that elderly subjects had significantly reduced saliva flow compared with young subjects. SIgA secretion rate is a function of absolute immunoglobulin concentration and saliva flow rate, and reflects the total amount of immunoglobulin available on the mucosal surface. ${ }^{26}$ Most of the enhancement in SIgA secretion rate can be accounted for by an increase in SIgA concentration in this study. Levels of SIgA in the elderly were significantly lower than in young healthy subjects (data not shown). Miletic et al ${ }^{29}$ also reported that SIgA secretion rates at rest were lower in the elderly than in young subjects. This reduced rate of SIgA secretion may explain the higher susceptibility of the elderly to infections.

In this study, we show that moderate exercise increased salivary SIgA levels. In contrast, it has been found that intense exercise training decreases salivary SIgA levels in young adults. ${ }^{561030}$ These phenomena may be related to the " $\mathrm{J}$ " curve hypothesis modelled for the relation between exercise and URTI.

At present, the underlying mechanism of enhanced salivary SIgA levels after 12 months of training in elderly subjects is not clear. It is generally accepted that the Thl cell subset becomes dominant relative to that of Th2 during aging. Exercise may result in more balanced Th1 and Th2 levels, whereby more immunoglobulins are being produced in local tissues. Although it is known that plasma concentrations of IgA and IgG increase with age, this may reflect decreased plasma volume. 


\section{Take home message}

SlgA levels in 45 elderly subjects had significantly increased after 12 months of exercise, indicating that regular moderate exercise enhances mucosal immune function in the elderly.

Our society is characterised by the growing number of elderly persons, who have a high risk of illness and infection such as URTI. Thus, there is a need for a simple method to evaluate immune function in order to design new strategies to improve such function..$^{29}$ Saliva is an easily retrievable sample material, which can be collected non-invasively. We emphasise that this study presents important results about only one aspect of the beneficial effects of exercise in the elderly, although we were unable to use a proper control group (untrained group or sham-trained group). Finally, we have shown here that exercise is a suitable strategy for improvement of mucosal immune function in the elderly.

\section{ACKNOWLEDGEMENTS}

We thank Keisuke Akimoto (Mitsubishi Heavy Industries, Ltd) for critical comment and review of the manuscript, and Fuminori Kimura, Kai Tanabe, Keisuke Koizumi, Keisuke Suito, Ryoji Nakamoto, Daisuke Nakamura, Manabu Otani, and Taku Soma (Department of Sports Medicine, Institute of Health \& Sport Sciences, University of Tsukuba) for their skilful assistance. This study was supported in part by a Grant-in-Aid for Scientific Research from the Ministry of Education, Science and Culture of Japan (13558003, 11680014), and a grant from the TARA Project of the University of Tsukuba.

\section{Authors' affiliations}

T Akimoto, Department of Life Sciences, Graduate School of Arts and Sciences, University of Tokyo, 3-8-1 Komaba, Meguro, 153-8902 Tokyo, Japan

T Akimoto, Y Kumai, T Akama, Japan Institute of Sports Sciences, Nishigaoka 3-15-1, Kita, $115-0056$ Tokyo, Japan

E Hayashi, I Kono, Department of Physical Therapy, Ibaraki Prefectural University of Health Sciences, Ami, 300-0394 Ibaraki, Japan

T Akama, Department of Sports and Health and Fitness, Faculty of

Physical Education, Japan Women's College of Physical Education, Kitakarasuyama 8-19-1, Setagaya, 157-8565 Tokyo, Japan H Murakami, R Soma, S Kuno, Tsukuba Advanced Research Alliance (TARA) Center, University of Tsukuba, Tsukuba, 305-8575 Ibaraki, Japan I Kono, Institute of Health and Sport Sciences, University of Tsukuba, Tsukuba, 305-8575 Ibaraki, Japan

\section{REFERENCES}

1 Shephard RJ, Shek PN. Exercise, aging and immune function. Int J Sports Med 1995;16:1-6.

2 Nieman DC, Henson DA, Gusewitch G, et al. Physical activity and immune function in elderly women. Med Sci Sports Exerc 1993:25:823-31.

3 Houston MS, Silverstein MD, Suman VJ. Risk factors for 30-day mortality in elderly patients with lower respiratory tract infection. Arch Intern Med $1997 ; 157: 2190-5$
4 Heath GW, Ford ES, Craven TE, et al. Exercise and the incidence of upper respiratory tract infections. Med Sci Sports Exerc 1991;23:152-7. 5 Mackinnon LT, Ginn EM, Seymour J. Temporal relationship between decreased salivary $\lg \mathrm{A}$ and upper respiratory tract infection in elite athletes. Australian Journal of Science, Medicine and Sports 1993:25:94-9.

6 Mackinnon LT, Hooper S. Mucosal (secretory) immune system responses to exercise of varying intensity and during overtraining. Int J Sports Med 1994; 15:S179-83.

7 Peters EM, Bateman ED. Respiratory tract infections: an epidemiological survey. S Afr Med J 1983;64:582-4.

8 Peters EM, Goetzsche JM, Grobbelaar B, et al. Vitamin C supplementation reduces the incidence of post-race symptoms of upper-respiratory-tract infection in ultra marathon runners. Am J Clin Nutr 1993;57:170-4.

9 Nieman DC. Exercise, upper respiratory tract infection, and the immune system. Med Sci Sports Exerc 1994;26:128-39.

10 Gleeson M, Mcdonald WA, Pyne DB, et al. Salivary lgA levels and infection risk in elite swimmers. Med Sci Sports Exerc 1999;31:67-73.

11 Tomasi TB. The discovery of secretory $\lg A$ and the mucosal immune system. Immunol Today 1992;13:416-18.

12 McDowell SL, Hughes RA, Housh RJ, et al. The effect of exercise training on salivary immunoglobulin $A$ and cortisol responses to maximal exercise. Int J Sports Med 1992;13:577-80.

13 Tomasi TB, Trudeau FB, Czerwinski D, et al. Immune parameters in athletes before and after strenuous exercise. J Clin Immunol 1982;2:173-8.

14 Chandler JM, Hadley EC. Exercise to improve physiologic and functional performance in old age. Clin Geriatr Med 1996;12:761-84.

15 Fiatarone MA, Marks EC, Ryan ND, et al. High-intensity strength training in nonagenarians. Effects on skeletal muscle. JAMA 1990;263:3029-34.

16 Green JS, Crouse SF. The effects of endurance training on functional capacity in the elderly: a meta-analysis. Med Sci Sports Exerc 1995;27:920-6.

17 Pedersen BK. Exercise immunology. 1st ed. Heidelberg: Springer-Verlag, 1997

18 Flynn MG, Fahlman M, Braun WA, et al. Effects of resistance training on selected indexes of immune function in elderly women. J Appl Physiol 1999:86:1905-13.

19 Mazzeo RS. The influence of exercise and aging on immune function. Med Sci Sports Exerc 1994;26:586-92

20 Shinkai S, Kohno H, Kimura K, et al. Physical activity and immune senescence in men. Med Sci Sports Exerc 1995;27:1516-26.

21 Crist DM, Mackinnon L, Tompson RF, et al. Physical exercise increases natural cellular mediated tumor cytotoxicity in elderly women. Gerontology 1989;35:66-71.

22 American College of Sports Medicine. Guidelines for exercise testing and prescription. 4th ed. Philadelphia: Lea \& Feibiger, 1991.

23 Karvonen MJ, Kentala E, Mustala $O$. The effects of training on heart rate: a longitudinal study. Ann Med Exp Biol Fenn 1957;35:307-15.

24 Shindo M, Kumagai S, Tanaka H. Physical work capacity and effect of endurance training in visually handicapped boys and young male adults. Eur J Appl Physiol 1987:56:501-7.

25 Akimoto T, Akama T, Sugiura K, et al. Alteration of local immunity in the oral cavity after endurance running. Japanese Journal of Physical Fitness and Sports Medicine 1998;47:53-62.

26 Mackinnon LT, Jenkins DG. Decreased salivary immunoglobulins after intense interval exercise before and after training. Med Sci Sports Exerc 1993;25:678-83

27 Nehlsen-Cannarella SL, Neiman DC, Balk-Lamberton AJ, et al. The effect of moderate exercise training on immune response. Med Sci Sports Exerc 1991;23:64-70.

28 Hanson LA, Biörkander J, Oxelius VA. Selective lgA deficiency. In: Chandra RK, ed. Primary and secondary immunodeficiency disorders. Edinburgh: Churchill Livingstone, 1983:62-4.

29 Miletic ID, Schiffman SS, Miletic VD, et al. Salivary lgA secretion rate in young and elderly persons. Physiol Behav 1996;60:243-8.

30 Akimoto T, Akama T, Koda Y, et al. Effect of repetitious intense exercise training on resting salivary $\lg \mathrm{A}$. Japanese Journal of Physical Fitness and Sports Medicine 1998;47:245-52. 